\title{
ESTABLISHING THE FEATURES OF A ROBOTIC ARM THEREBY DETERMINING THE PARAMETERS FOR ITS MOTION AND VIBRATION STABILITY
}

Akannyene, M. Joshua Ph.D. ${ }^{1}$, Prof. Eneh Ifeanyi I. ${ }^{2}$ andUche, Okafor $\mathbf{P}^{3}$ Department of Electrical and Electronic Engineering, Faculty of Engineering, Enugu State University of Science Technology (ESUT).

The need to establish the features of a robotic arm is of importance to the control engineer. This is because it will enable him to know the best application to which the robot could be applied. Characterization is then carried out in order to determine the capabilities of the manipulator and the effectors. In this work, the characterization considered the degree of freedom, possible motion parameters and vibration stability. Motion parameterization is important to determine the area of application of the robotic arm. Similarly, vibration stability is related to its precision and accuracy in carrying out the desired operation. Without proper establishment of the features of the robotic arm, its application in industrial processes cannot be guaranteed. The mechanisms of its operations and other relevant features are dependent to a large extent on a successful characterization. Future work the robotic arm will depend on the result of its characterized features.

Keyword: Robotic Arm, Characterization, Parameterization, Manipulator, Effector, Motion, Vibration Stability

\subsection{Introduction}

Most existing robotic manipulators are designed with maximum stiffness, in an attempt to minimize system vibration and achieve good positional accuracy.As a consequence, such robots are usually heavy with respect to the operating payload. This, in turn, limits the speed of operation of the robot manipulation, increases the size of actuator, boosts energy consumption and increases the overall cost. The study of robot manipulator mechanisms and motion has therefore become indispensable. Mathematically, the motion of a robot manipulator can be computed through the integration of kinematics (forward and inverse kinematics), dynamics and trajectory planning calculations. However, it is difficult to apply these mathematical solutions. Problems include failure to derive the kinematic equations and inability to find analytical solutions or to solve the variety of complex mathematical equations. Furthermore, the mathematical method can only be applied if the characteristics of the trajectories are known. It is a complex and tedious process to compute the motion of a robot manipulator by using the conventional approach.A typical flexible manipulator system has a flexible link, an actuator-gear mechanism to rotate the link, an optical encoder to measure joint rotation, accelerometers and strain gauges to sense flexible motion, an optical arrangement to measure the endpoint position and an occasional force sensor attached to the end-point.Research on flexible manipulators has advanced towards two link and multilink systems. A multilink manipulator has two or more joints making it capable to move in all directions. It is important for control purposes to recognize the flexible nature of the manipulator system and to build a suitable mathematical framework for modeling and controlling of the system. The basic applications of these flexible manipulators can be seen in robotic arm system such as Pick and Place robot arm used in picking objects from one 
location to another. The classical controllers have been used by researchers to control motion, noise and vibrations like proportional $(\mathrm{P})$, proportional derivative (P-D), proportional integral derivative (P-I-D), and so forth. But these have many shortcomings and are not as efficient as intelligent controllers.

End effector could be a tool and its fixture or gripper or any other device. Just like the fingers, the grippers are generally used in grasping and holding an object from a point to a desired location. Grippers are classified as mechanical gripperslike vacuum or suction cups, magnetic grippers, adhesive grippers, hooks, scoops, etc. For the Tools; a robot is required to manipulate a tool to carry out an operation on a work part. Here, the tool acts as end- effector. There are many types of tool depending on the type of action, task or function to be performed. Some examples of used end-effectors include; spot-welding tools, arc-welding tools, nozzles for spray-painting, and rotating spindles for drilling and grinding and so on.

\subsection{Review of Related Works}

Different author have carried out researches on motion and stability of robots. Their works have provided requisite knowledge and insight into robotic manipulators leading to their motion and stability. Reviews of the works of these authors are presented here accordingly. According to Ramesh et al, (2016), in Design of a 3 DoF Robotic Arm, the degree of redundancy determines the manipulability and dexterity of any robotic manipulator. Due to its simplistic design, serial robotic arm is very popular in industrial applications. The paper treated the design of three degree of freedom of a serial robotic arm, using CAD to develop its mechanical structure. The dynamic properties of the robotic arm were analyzed adopting Euler-Lagrange method in the development of the robotic arm complex equation of motion. Using RoboAnalyzer@ software in the simulation and comparing with the analytical result, there was an agreement between the two.

Farman et al, (2018) presented a paper titled, "Design of a Three Degrees of Freedom Robotic Arm."A three degree of freedom robotic arm design, expected to pick and place light weight objects based on colour sorting mechanism, was presented. It was made of three joints namely, a gripper, two rectangular shaped links, a rotary table and a rectangular platform. While a servomotor powered the angular rotation of each joint, each servomotor shaft has its angular position controlled by an Arduino microcontroller signal used to execute a MATLAB code.Several static and dynamic calculations, mechanical properties calculations and prototype testing were presented in order to provide a final product with well-established structure and functionalities.

Kumar et al (2017) did a work titled, "Robotic Arm Movement Optimization Using Soft Computing." The paper opined that robotic use in industries is a commonplace due its efficiency and versatility. Furthermore, the optimization of robotic arm has attracted increased research of recent. Soft computing technique as an optimal control approach was proposed for movement and trajectory planning involving various degrees of freedom if a robotic arm. Uncertainties such as movement, friction and settling time in the robotic arm movement were evaluated and analyzed.Its kinematics behaviour needed to be understood before optimization. The optimization was done using genetic algorithm and the model joints. Simulation showed satisfactory results as target position was approached within the simulation time.

The reviewed related works were carried as a precursor to the establishment of the features as expected in this discourse.

\subsection{Methodology}

Establishing the features of a robotic armis to characterize an existing robot arm to determine the parameters that affect its motion and vibration. An existing automobile industrial flexible 
manipulator, employed by Mitsubishi Automobile (PA10 Manipulator (robotic arm)) was used, see figure 3.1. The system was developed by (Ngoc et al., 2009) using a custom PID controller for critical real time performance, using QNX 6.3 operating system.

The characterization was performed considering various parameters such as the respective joints of the manipulator, the links, inertia, trajectory, torque, cost function and the input vector $(\mathrm{X})$, with the aim of identifying the robot joint parameters, linearized results of the custom controller and rigid body dynamics.

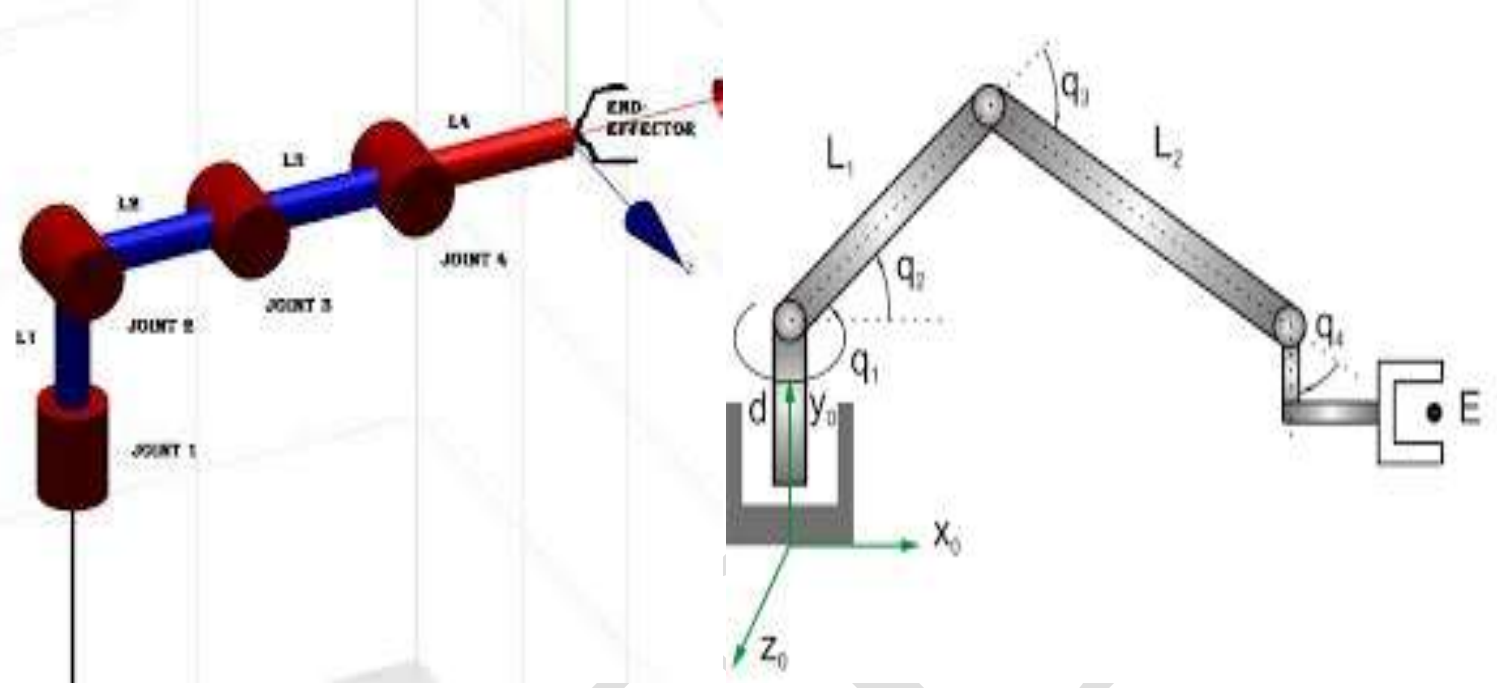

Figure 3.1: Mitsubishi PA10 Manipulator (Ngoc et al., 2009)

The manipulator in view consists of four joints of which their boundary velocity is analyzed respectively according to the experiment carried out by Ngoc et al., (2009) as shown in table 3.1: The joint toque was obtained by measuring the motor current.

Table 3.1: Boundary velocity for each joints of the manipulator (Ngoc et al., 2009)

\begin{tabular}{|l|l|}
\hline Joint & $\begin{array}{l}\text { Boundary velocity }(\mathbf{q}(\mathrm{rad} / \mathbf{s})), \\
\text { where } \mathbf{q}=\text { angular joint in } \mathbf{r a d} / \mathbf{s})\end{array}$ \\
\hline 1 & 0.25 \\
\hline 3 & 0.27 \\
\hline 4 & 0.3 \\
\hline
\end{tabular}

The inertia was analyzed by dynamic modeling of the manipulator, considering the degree of freedom, vectors of the joints, velocity, position, inertia matrix, Coriolis-centrifugal, gravity vector in the joint space and the vector force at each of the joints (Eke James. 2004). These factors are linearized for identification and parameterization purpose taking into account the Denavit Hartenberg (DH) parameters consisting of XX, XY, XZ, YY, YZ, ZZ, Mx, My, Mz, $\mathrm{Mi}$; where the XX, XY, XZ, YY, YZ, ZZ are the inertia tensor of the link and the Mx, My, $\mathrm{Mz}, \mathrm{Mi}$ are the moment and the link mass.

The characterized results wereobtained (table 3.2) using the idea of virtual parameters and constraint optimization tool proposed by Mata et al., (2015) to determine any manipulator of unknown inertia, considering the input vector parameters like the standard dynamics of the links and the cost function which defines the least square solution. Clearly, this presents a set of physically feasible parameters that will guide further advancement in the design.On a general note and basically too, for any rotating object, the moment of inertia can be calculated by taking the distance of each particle from the axis of rotation ( $r$ in the equation), squaring that value (that's the $r^{2}$ term), and multiplying it times the mass of that particle. You 
do this for all of the particles that make up the rotating object and then add those values together, and that gives the moment of inertia. The following formula applies:

$I_{p}=\sum_{i=1}^{N} m_{1} r_{i}^{2}$

The consequence of this formula is that the same objects gets a different moment of inertia value depending on how it is rotating. This means that even if the physical shape of the object remains the same a new axis of rotation ends up with a different formula,

Table 3.2: characterized result of the manipulator joint inertia (Mata et al., 2015)

\begin{tabular}{|c|c|c|c|c|}
\hline DH Parameters & Joints 1[Kg-m²] & Joint 2[Kg-m $\left.{ }^{2}\right]$ & Joint 3[Kg-m $\left.{ }^{2}\right]$ & Joint $4\left[\mathrm{Kg}-\mathrm{m}^{2}\right]$ \\
\hline $\mathbf{X X}$ (Inertia tensor) & 0.3811 & 0.0347 & 0.0236 & 0.0758 \\
\hline $\mathbf{Y Y}_{(\text {Inertia tensor) }}$ & 0.6884 & 1.8651 & 0.0119 & 0.0073 \\
\hline $\mathbf{Z Z}$ (Inertia tensor) & 0.0356 & 3.006 & 0.1705 & 0.544 \\
\hline $\mathbf{X Y}$ (Inertia tensor) & 0.9833 & 0.7599 & 0.5881 & 0.8631 \\
\hline $\mathrm{XZ}_{\text {(Inertia tensor) }}$ & 1.1198 & 0.8079 & 0.5955 & 0.1214 \\
\hline $\mathbf{Y Z}$ (Inertia tensor) & 0.317 & 0.0001 & 1.5338 & 0.019 \\
\hline $\begin{array}{l}\text { Mx(Moment.link } \\
\text { mass) }\end{array}$ & 0.8353 & 2.3296 & 1.3338 & 0.8852 \\
\hline $\begin{array}{l}\text { My(Moment.link } \\
\text { mass) }\end{array}$ & 0.8831 & 0.0827 & 0.4072 & 0.1501 \\
\hline $\begin{array}{l}\text { Mz(Moment.link } \\
\text { mass) }\end{array}$ & 3.1872 & 0.4506 & 0.2006 & 1.6751 \\
\hline $\begin{array}{l}\text { Mi(Moment.link } \\
\text { mass) }\end{array}$ & 0.4696 & 0.2088 & 2.5769 & 3.7324 \\
\hline
\end{tabular}

The data in table 3.2 is the characterized result of the manipulator joint inertia.

\subsection{Results and Analysis}

Simulation was carried out on the features established in this work. This shows their dynamic behaviours during operations in industrial applications. Their dexterity during use are also demonstrated in the analysis as shown.

Simulation was carried out using models of Figures 3.13 and 3.37. Also, the simulation parameters were presented in table 4.1.The purpose of this simulation was to determine accuracy and time-response of the developed systems. According to Okafor et al., (2017), time response is the time behavior of the system when its input was changed from zero to a positive or negative value. The essence of this is to determine the stability of the developed system and its ability to reach one stationary state when starting from another. The manipulator (robot arm) was excited with step input with consideration of voltage and current rating.

Table 4.1: Simulation parameters

\begin{tabular}{|l|l|}
\hline Number of controller & 6 \\
\hline Control sampling time & $10 \mathrm{~s}$ \\
\hline Frequency & $10 \mathrm{~Hz}$ \\
\hline Total number of dc motors & 9 \\
\hline Number of joints & 6 \\
\hline input voltage & $\pm 5 \mathrm{~V}$ \\
\hline
\end{tabular}

To evaluate the performance of the robot arm, it is paramount to evaluate the linearization of the various joints of the manipulator. From the simulation result, Figure 4.6 showed the resultant effect of the PID controller linearized for each joints. It can be observed from the 
figure that there are lots of variations along the linearized dynamics of the six joints (Eneh I. I., 2012).

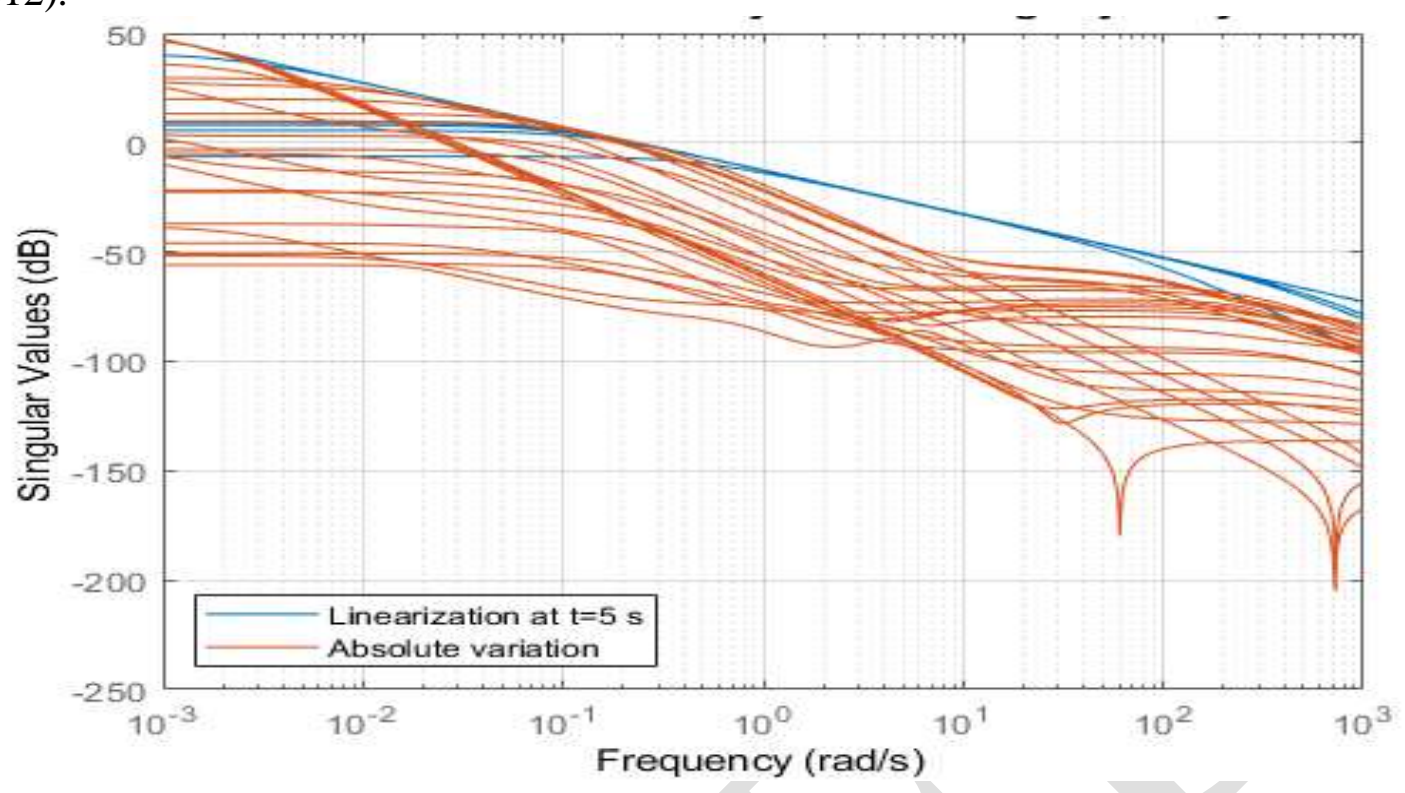

Figure 4.1: Variation of linearized dynamics along trajectory

The gain was varied and system response is as shown in Figure 4.2. It could also be seen that from Figure 4.3, that there are still signs of variations in the signal and needs to be eradicated.

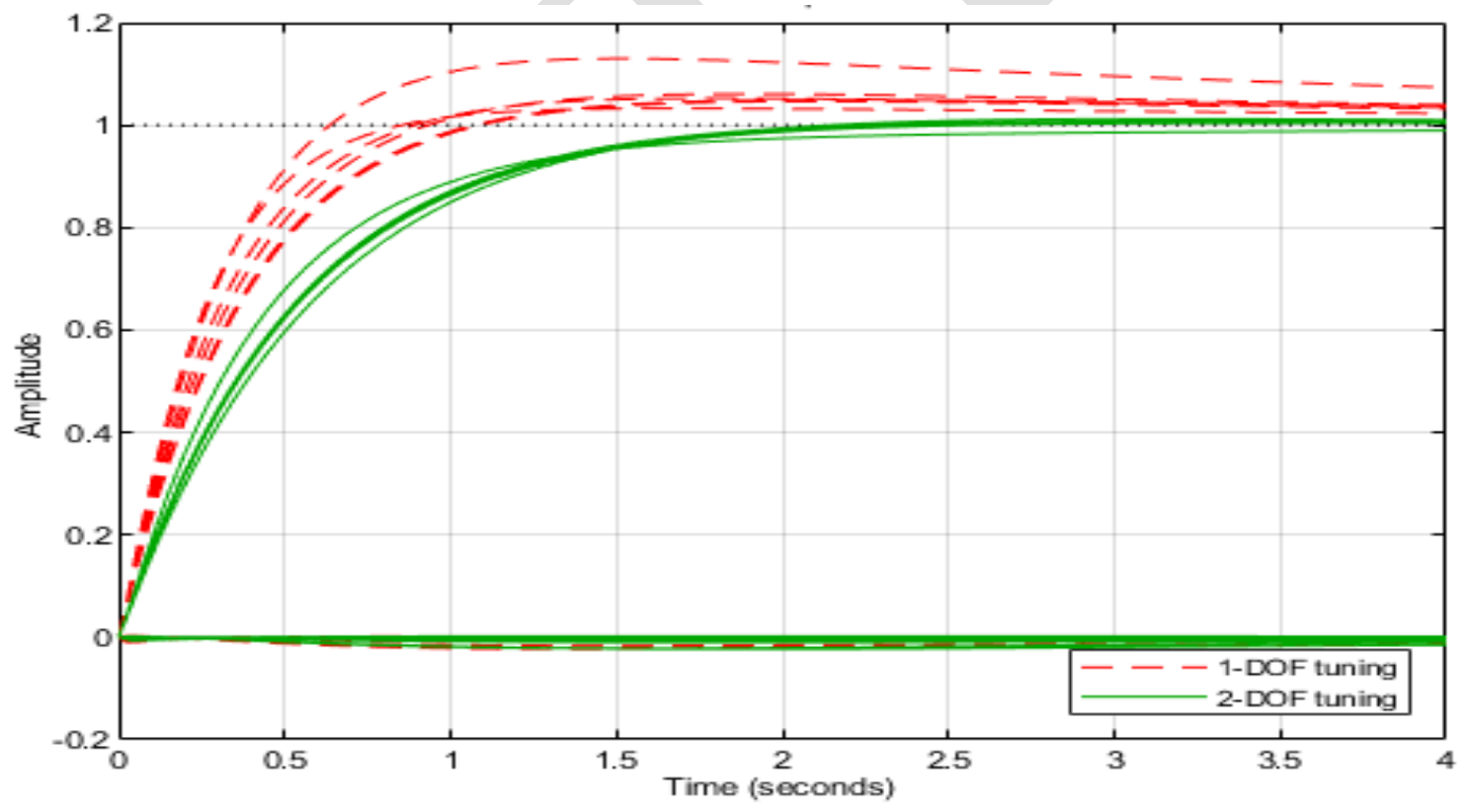

4.2: Tuned response of the PID controller

Those variations in signal are what translates to oscillations and finally to mechanical vibration. So, the PID system was further fine-tuned to produce the resultant effect as shown in figure 4.3. 


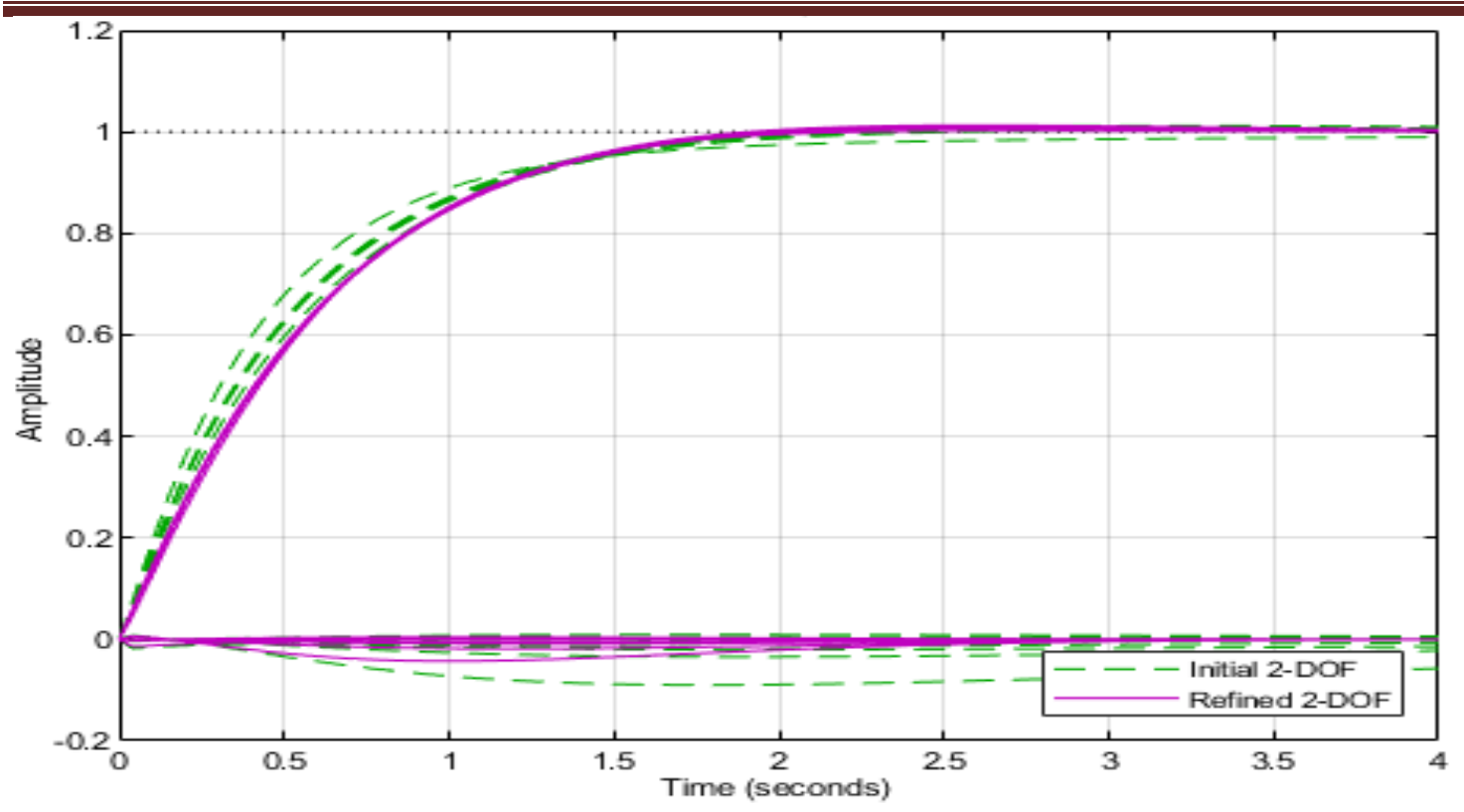

Figure 4.3: Fine tuning response of the manipulator

The set point tracking result for the tuned PID controller is presented in figure 4.4; showing the relative error (disturbance rejection analysis) at various amplitude signal, it can be seen that the tracking patterns (green) is not very accurate at certain times (sec). The accumulation of error brings inaccuracy in the speed of the manipulator thereby, affecting its motion. In this case, the system experienced overshot because the PID controller could not adapt to the target voltage of the system.

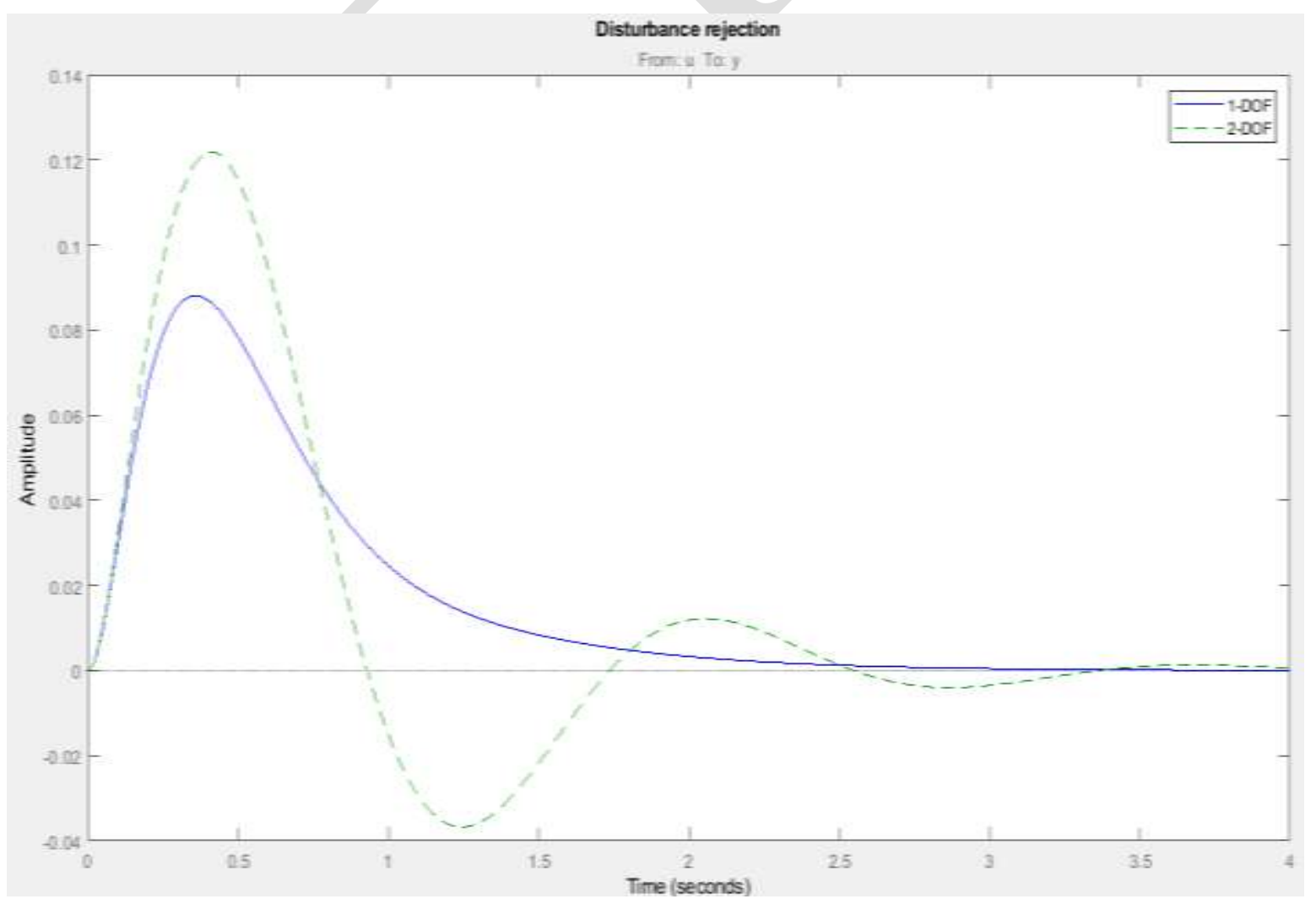

Figure 4.4: Tuned PID controller result 
Now, what is needed is an adaptive controller that can make the target (output) voltage to follow the reference (input) voltage. However the neural network controller has the capability to compensate for the voltage overshoots and ensure system stability. So, the hybrid controller was used instead in this case to handle that nonlinear nature of the robot arm and a linearized response for the manipulator is as shown in figure 4.5.

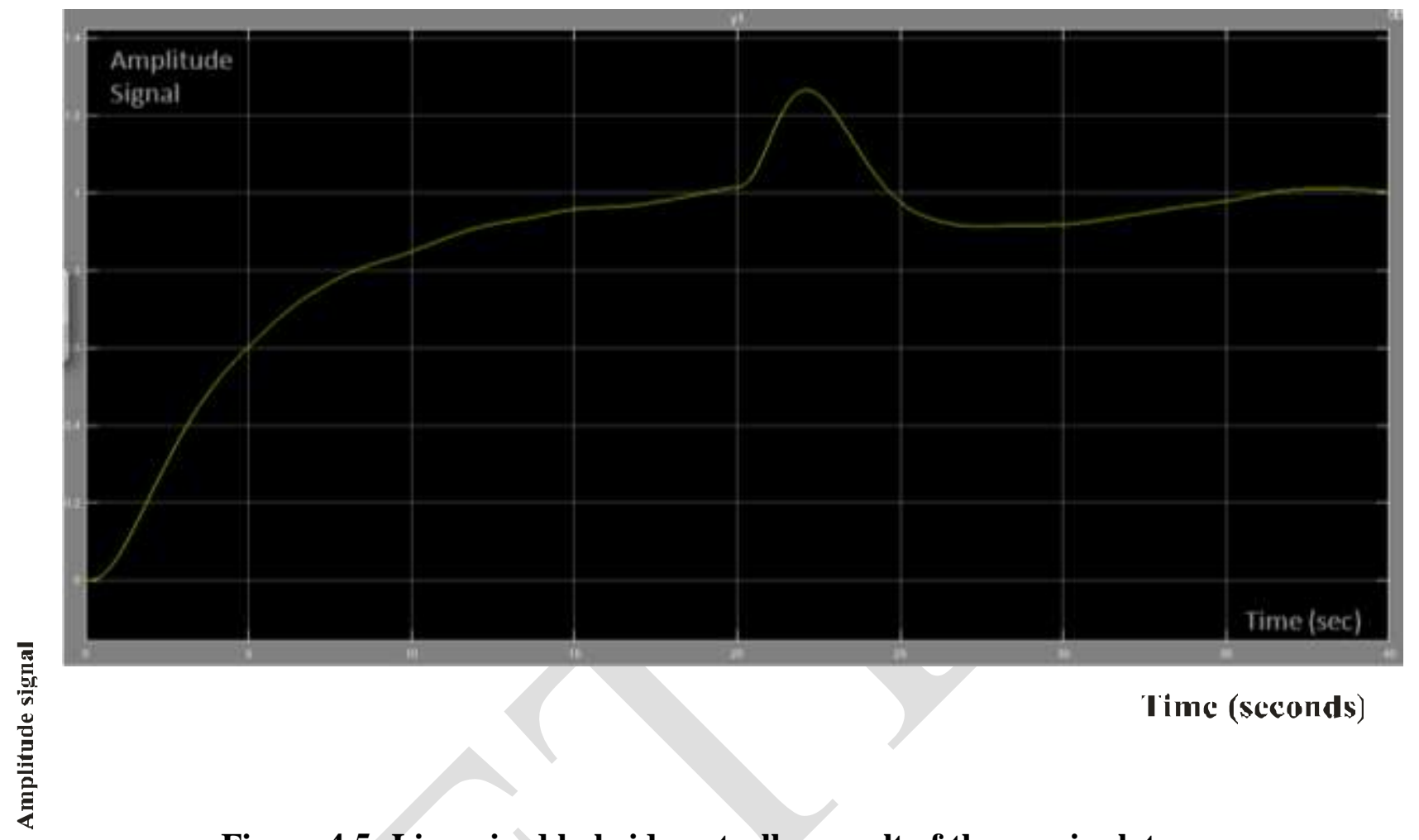

Figure 4.5: Linearized hybrid controller result of the manipulator

The dynamic responses shown in Figures 4.1 to 4.5 are the robotic arm behaviours given normal operational applications to enhance higher efficiency and improved output. However, optimal results are obtained when robots are trained in a controlled working condition.

\subsection{Conclusion}

In order to achieve high performance in target position and movements, high level of precision is paramount and of critical importance in the control of motion and vibration of any manipulator, especially in Pick and Place robot arm. Also, it should be noted that control of automated processes, is not a simple task because, real-time processes mostly exhibit nonlinear behavior such as overshoot, dead zone, saturation, backlash, coulomb friction, and delays between the system inputs and outputs. Then, the issue is how best to minimize or bring down tracking error of the controller to zero or to a minimally acceptable level within the shortest interval in order to achieve higher precision in target position and movement of manipulators. All these phenomenal developments are direct results of other factors which such as environmental operation, industrial application and training given to the robotic effectors.

\section{References}

1. Ramish, Syed Baqar Hussain and Farah Kanwal (2016), Design of a 3 DoF Robotic Arm, Conference Paper,2016 Sixth International Conference on Innovative Computing Technology (INTECH). 
2. Madiha Farman, Muneera Al-Shaibah, Zoha Aoraiath and Firas Jarrar, (2018), Design of a Three Degrees of Freedom Robotic Arm, International Journal of Computer Applications (0975 - 8887), Volume 179 - No.37, April 2018.

3. Surender Kumar1, Kavita Rani2, and V. K. Banga, (2017), Robotic Arm Movement Optimization Using Soft Computing, International Journal of Robotics and Automation (IJRA) Vol. 6, No. 1, March 2017, pp. 1 14.

4. Cristóvão Jorge Silva Duarte Sousa (2014); Dynamic model identification of robot manipulators: Solving the physical feasibility problem; University Of Coimbra.

5. D'Azzo, J.,Abdallah, C., Dawson, D., Dorato, P. and Jamshidi, M. (1991) Survey of robust control for rigid robots, IEEE Control Systems Magazines, pp.24-30

6. Dorf, R.C. and Bishop, R.H. (2008) Modern Control Systems, Pearson Prentice Hall, 11th Ed.

7. Hägglund T., and Åström K. J., (2004), "Revisiting the Ziegler-Nichols Tuning Rules for Pi Control : The Frequency Response Method"; Asian Journal of Control, Vol. 6, No. 4, pp. 469-482.

8. Kadu C.B., (2015), "Auto-tuning of PID Controller for Robot Arm and Magnet Levitation Plant": International Journal of Research in Engineering and Technology.

9. Kumar, R.G.U.V., and Raja, C.V.N. (2014) Comparison between FSC and PID Controller for

10. 5DOF Robot Arm, International Journal of Emerging Trends in Electrical and Electronics (IJETEE - ISSN: 2320-9569), Vol. 10, Issue. 2, pp.1-6.

11. Pranaya R.B. (2017); force / position robot manipulator control using the PID controller, India 